\title{
Dynamic analysis of tension cable using degenerated solid beam element
}

\author{
Duzheng Shi \\ Henan Transportation Research Institute CO., LTD, China \\ Research and Development Center on Bridge Safety Detection \& Reinforcement Technology, \\ Ministry of Transport, China
}

\begin{abstract}
KEYWORD: finite element; degenerated solid beam element; natural vibration
ABSTRACT: Based on spatial elasticity theory, the fundamental beam assumptions are introduced into the 20-node spatial isoparametric finite element that constructing the degenerated solid beam element in order to overcome the simulation limitations of the beam element on its cross-section scale. According to the numerical examples about the natural vibration analysis of cables, the results show the degenerated solid beam element has the effectiveness and stability precision. Moreover, the element can also be used to analyze the vibration of the varied cross section cable and some cable in the complex three-dimensional boundary.
\end{abstract}

\section{INTRODUCTION}

Tension cables are critical structural components in modern civil engineering, and have been widely used. It plays different roles in the structure, for example, stay cables in cable-stayed bridges, main cables and sling in cable-suspension bridge, suspender in arch bridges and external prestressing cable in strengthened bridge. In addition, cables as an important component part of buildings, bear loads in large-span roof and tensegrity structure. Owing to their large flexibility, relatively small mass and extremely low damping, stay cables presents a significant differences with beam or struts components in the mechanical performance.

In general, monitoring cable tensions during the construction of cable-supported bridges is necessary to align cables properly and to ensure no cable is overloaded. After the completion of the bridge, cables serving as the primary vertical load carrying elements of the bridge, there is a need to ensure the structural integrity of the cables well. Furthermore, small variations in cable tension may cause a dramatic effect on the global response of other parts of the bridge including the deck and pylon. The exact estimation of the cable tension force has been the main objective of bridge maintenance. For this purpose, some methods have been developed for measuring the tension in a bridge cable. The simple estimation formulas used in the vibration method for the bridge cable can be derived from the transverse vibrations of a taut string with the assumption of no sag. However, this current method has some limitations, because bridge cables always don't behave as taut strings because of their flexural rigidities. In particular, to estimate the tension of shorter cables that are greatly influenced by flexural rigidity.

Therefore, some new methods are proposed that are based on the finite element model of the cable. The nonlinear characteristic and the complicated engineering conditions can be considered in the model. However, the current elements as one dimensional link, pipe and beam based on the assumption that the section is plane, cannot be satisfied to simulate the distribution of rigid stiffness and mass distribution of the real bridge cable. In this paper, a new approach is developed to model the cable dynamic characteristic with the degenerated beam element, which is modified by the 20node spatial isoparametric finite element based on the basic assumptions of beams.

\section{BASIC THEORIES AND METHODS}

Bridge cables are composed with steel strands, protective cover and filler. Therefore, the real performance of the cable is different with the computing model. Degenerated solid elements as a new 
spatial analysis mode were developed by Pro. Xu at Zhejiang University. Compared with the usual three dimensional solid isoparametric element, it has the same discrete form, shape function, balance and geometric equation.

The current beam and link element was developed to show the general mechanical performance of the structure on the classic elastic theory. In the degenerated solid elements, the current assumption can be introduced to expand the elastic matrix, and then obtain the three-dimensional isoparametric element form of the beam components. As the real cable section is various, 20-nodes hexahedron isoparametric element can approximate the irregular boundaries and get a well calculation accuracy.

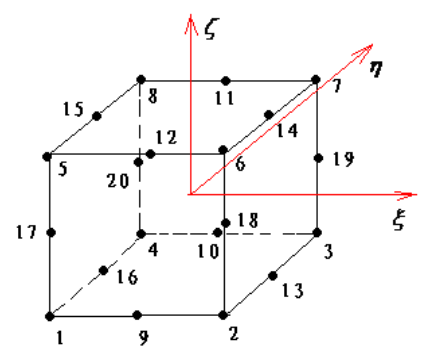

Figure 1. displacement vector of the 20-nodes isoparametric element.

$\{d\}^{e}=\left[\begin{array}{lllll}\delta_{1} & \delta_{2} & \delta_{3} & \mathbf{L} & \delta_{20}\end{array}\right]^{T}=\left[\begin{array}{llllllllll}u_{1} & v_{1} & w_{1} & u_{2} & v_{2} & w_{2} & \mathbf{L} & u_{20} & v_{20} & w_{20}\end{array}\right]^{T}$

The shape function of the 20-nodes isoparametric element

$N_{i}=\bar{N}_{i}-\sum_{j=1}^{12} \bar{N}_{j}\left(\xi_{j+8}, \eta_{j+8}, \zeta_{j+8}\right) N_{j+8}$

$\left.\bar{N}_{i}=\left(1+\xi_{i} \xi\right)\left(1+\eta_{i} \eta\right)\left(1+\zeta_{i} \zeta\right) / 8 \quad{ }_{i=1,2, \mathrm{~L} 8}\right)$

$N_{i}=\left(1-\xi^{2}\right)\left(1+\eta_{i} \eta\right)\left(1+\zeta_{i} \zeta\right) / 4 \quad(i=9,10,11,12)$

$N_{i}=\left(1-\eta^{2}\right)\left(1+\xi_{i} \xi\right)\left(1+\zeta_{i} \zeta\right) / 4 \quad(i=13,14,15,16)$

According to the spatial elastic theory, the stress-strain relationship of a solid element can be described as, and the constitutive relationship model $D$ is

$[D]=\left[\begin{array}{llllll}d_{1} & & & & & \\ d_{2} & d_{1} & & & & \\ d_{2} & d_{2} & d_{1} & & & \\ & & & d_{3} & & \\ & & & & d_{3} & \\ & & & & & d_{3}\end{array}\right]$

where $d_{1}=\frac{E(1-\mu)}{(1+\mu)(1-2 \mu)} \quad d_{2}=\frac{\mu}{1-\mu} d_{1} \quad d_{3}=\frac{E}{2(1+\mu)}, E$ is Young's modulus, and $\mu$ is Poisson's ratio.

After introducing the assumption that the transverse stress is very small than other stress and strain caused by is can be neglected, the elastic matrix of the degenerated solid element can be revised as 
$D=\left[\begin{array}{cccccc}E & 0 & 0 & 0 & 0 & 0 \\ 0 & \lambda E & 0 & 0 & 0 & 0 \\ 0 & 0 & \lambda E & 0 & 0 & 0 \\ 0 & 0 & 0 & d_{3} & 0 & 0 \\ 0 & 0 & 0 & 0 & d_{3} & 0 \\ 0 & 0 & 0 & 0 & 0 & d_{3}\end{array}\right]$

where $\lambda$ is penalty parameter $(\lambda=1000)$ to meet the mechanical performance of beam element, and have a same order as the spatial isoparametric element.

Compared with the current element, the degenerate element can be divided into several regions, with each region having its own material, and the cavity in the element is treated as a special kind of material. The stiffness matrix of the new parent element is integrated by different regions composed with 8-20 nodes. The region nodes can be determined by the coordinates in the parent element. Given the local coordinates of node $i$ in the region $k$ as $\left(\varepsilon_{i}^{k}, \eta_{i}^{k}, \zeta_{i}^{k}\right)$, any the coordinates of any point in the region can be described as

$\varepsilon^{k}=\sum_{i=1}^{n m} N_{i}\left(\varepsilon^{\prime}, \eta^{\prime}, \zeta^{\prime}\right) \varepsilon_{i}^{k}$

$\eta^{k}=\sum_{i=1}^{n m} N_{i}\left(\varepsilon^{\prime}, \eta^{\prime}, \zeta^{\prime}\right) \eta_{i}^{k}$

$\zeta^{k}=\sum_{i=1}^{n m} N_{i}\left(\varepsilon^{\prime}, \eta^{\prime}, \zeta^{\prime}\right) \zeta_{i}^{k}$

where $m$ is the number of regions in the element; Dk is the material elastic matrix; $\rho^{k}$ is the density of the region $\mathrm{k} ; J^{\prime} \mid$ is the Jacobi determinant of the region coordinate transformation matrix. Then the global equilibrium equation is solved by introduction the nodal force vector and boundary condition.

\section{SIMULATION OF THE DEGENERATE SOLID ELEMENT}

The cable model using the presented element was conducted to validity its effect by the VEAP program. The cable was meshed by the 20 -nodes degenerate solid element. The $4 \mathrm{~m}$ and $40 \mathrm{~m}$ cable had 580 nodes in 100 elements, while the $10 \mathrm{~m}$ and $100 \mathrm{~m}$ cable had 720 nodes in 125 elements. The constraints was applied on the two ends in the 3 degrees, and the uniform axial force was imposed along the cable.

Also the dynamic analysis by 2 -nodes beam element was carried out to contrast the results. 20 elements was meshed in both $4 \mathrm{~m}$ and $10 \mathrm{~m}$ cable while 40 elements in another two models. The hinged-hinged constraint conditions were used in the calculation model, and the axial force was simulated by initial strain.

In the two calculation model above, the diameter of the cable is $0.08 \mathrm{~m}$; The elastic module is $1.9 \times 10^{5} \mathrm{MPa}$; The Poisson's ratio is 0.167 ; The density is $8000 \mathrm{~kg} / \mathrm{m}^{3}$. The tension force on every cables changes from $400 \mathrm{kN}$ to $5000 \mathrm{kN}$. Fig. 2 shows the results from every calculation model. The graph's horizontal coordinate shows the cable force range, and the vertical coordinate shows the frequency of the cables. 


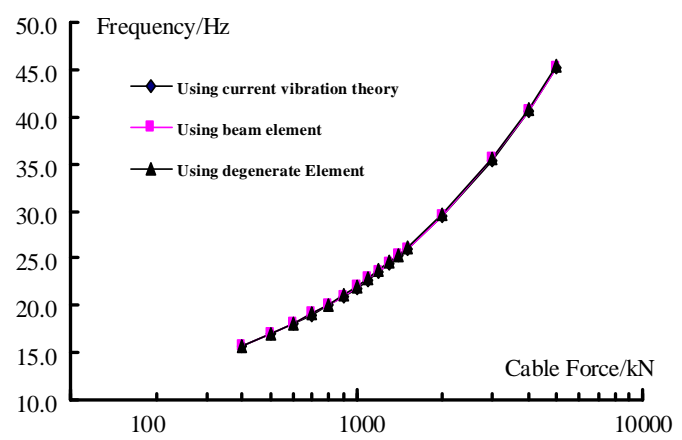

(a)

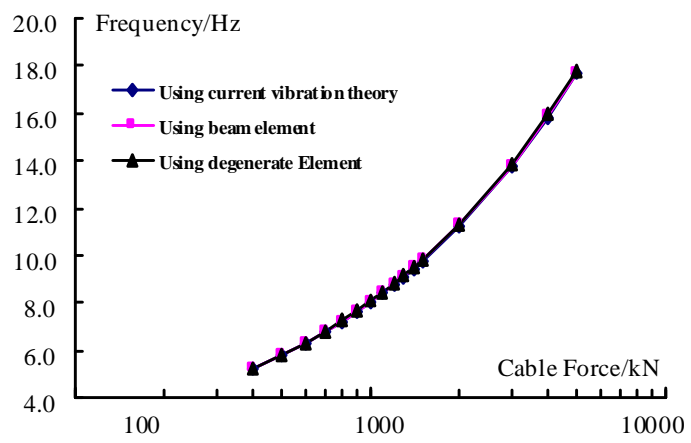

(b)

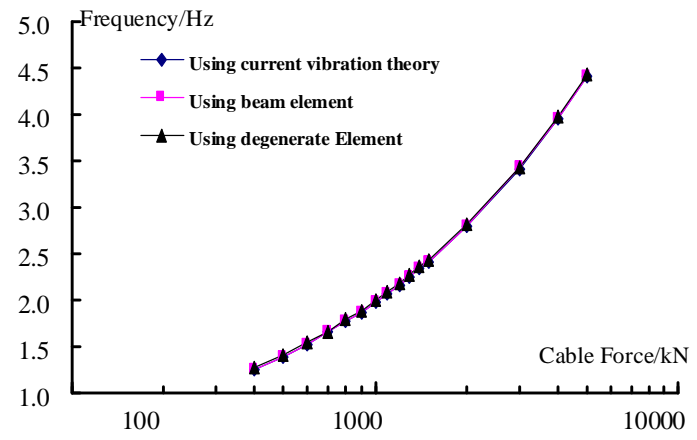

(c)

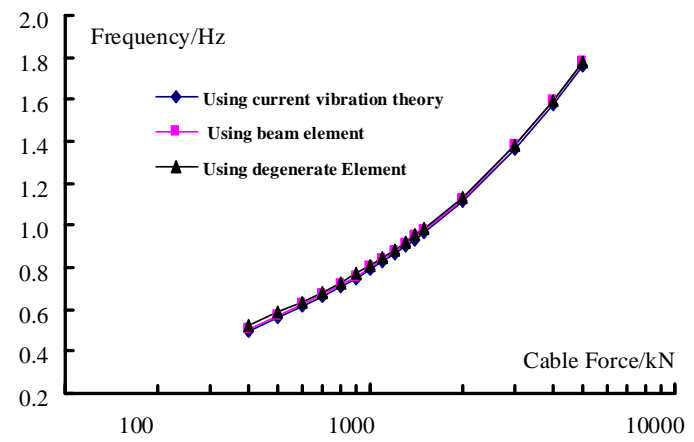

(d)

Figure 2. Contrast with frequencies of cables using different calculation models(a:40m cable; b:10m cable; c: $40 \mathrm{~m}$ cable; d: $100 \mathrm{~m}$ cable) 
Table 1. Calculated frequencies of cables under different axial force (A: Using degenerate Element ; B: Using beam element; C: Using current vibration theory)

\begin{tabular}{ccccccccccccc}
\hline $\begin{array}{c}\text { Cable } \\
\text { Force/kN }\end{array}$ & A & 4m Cable & B & C & A & 10m Cable & \multicolumn{3}{c}{ 40m Cable } & \multicolumn{3}{c}{ 100m Cable } \\
\hline 400 & 15.754 & 15.717 & 15.716 & 5.248 & 5.218 & 5.217 & 1.268 & 1.251 & 1.250 & 0.527 & 0.499 & 0.499 \\
500 & 16.958 & 16.909 & 16.907 & 5.817 & 5.783 & 5.782 & 1.415 & 1.398 & 1.397 & 0.584 & 0.558 & 0.558 \\
600 & 18.081 & 18.023 & 18.019 & 6.336 & 6.298 & 6.297 & 1.548 & 1.530 & 1.530 & 0.635 & 0.611 & 0.611 \\
700 & 19.139 & 19.072 & 19.067 & 6.815 & 6.775 & 6.772 & 1.670 & 1.653 & 1.652 & 0.683 & 0.670 & 0.660 \\
800 & 20.141 & 20.066 & 20.060 & 7.262 & 7.220 & 7.217 & 1.784 & 1.766 & 1.766 & 0.728 & 0.716 & 0.705 \\
900 & 21.095 & 21.014 & 21.006 & 7.683 & 7.639 & 7.635 & 1.891 & 1.873 & 1.872 & 0.770 & 0.758 & 0.748 \\
1000 & 22.009 & 21.921 & 21.912 & 8.083 & 8.036 & 8.032 & 1.993 & 1.975 & 1.974 & 0.810 & 0.799 & 0.788 \\
1100 & 22.885 & 22.791 & 22.781 & 8.463 & 8.419 & 8.410 & 2.089 & 2.071 & 2.070 & 0.848 & 0.837 & 0.827 \\
1200 & 23.730 & 23.631 & 23.619 & 8.828 & 8.777 & 8.772 & 2.181 & 2.163 & 2.161 & 0.884 & 0.874 & 0.864 \\
1300 & 24.545 & 24.441 & 24.427 & 9.178 & 9.126 & 9.119 & 2.270 & 2.251 & 2.250 & 0.919 & 0.910 & 0.899 \\
1400 & 25.334 & 25.225 & 25.210 & 9.514 & 9.461 & 9.454 & 2.355 & 2.336 & 2.334 & 0.953 & 0.944 & 0.933 \\
1500 & 26.099 & 25.987 & 25.969 & 9.840 & 9.785 & 9.777 & 2.437 & 2.418 & 2.416 & 0.985 & 0.976 & 0.966 \\
2000 & 29.631 & 29.501 & 29.474 & 11.327 & 11.267 & 11.255 & 2.812 & 2.792 & 2.789 & 1.134 & 1.126 & 1.115 \\
3000 & 35.659 & 35.509 & 35.458 & 13.831 & 13.764 & 13.742 & 3.441 & 3.421 & 3.416 & 1.384 & 1.378 & 1.366 \\
4000 & 40.805 & 40.649 & 40.569 & 15.946 & 15.877 & 15.844 & 3.972 & 3.952 & 3.944 & 1.595 & 1.590 & 1.577 \\
5000 & 45.372 & 45.217 & 45.104 & 17.812 & 17.743 & 17.697 & 4.440 & 4.420 & 4.409 & 1.782 & 1.778 & 1.763 \\
\hline
\end{tabular}

\section{CONCLUSION}

This paper presents a new element for the dynamic analysis of the tension cables, and a numerical example is given to study its validity. The results can be obtained as follows.

(1) The calculation frequency of different cables with degeneration element are basically consistent with theoretical and beam element solution. The result from the degeneration element model of $4 \mathrm{~m}$ cable is $0.242 \%$ higher than that from the theoretical solution, while $0.234 \%$ higher than from the beam element model. For the $10 \mathrm{~m}$ cable, this extension becomes $0.606 \%$ and $0.587 \%$. For $40 \mathrm{~m}$ cable, it becomes $1.448 \%$ and $1.446 \%$ respectively. It can be found that this deviation increase with the length of the cables. Up to $100 \mathrm{~m}$ cable, the deviation change into $5.589 \%$ and $3.574 \%$ respectively.

(2) Compared with the theoretical solution, the maximum relative error of the numerical results using beam element is not more than $2.8 \%$ in long cables. It might by caused by the effects of sag, which has a significant effect when cables become longer. In the basic dynamic theory, this effect is neglected in the vibration equation of small deflection beams.

(3) In the degenerated three-dimensional solid element, the spatial characteristic of the plane beam element is expressed by introducing an appropriate penalty coefficient in the elastic matrix, then the torsion and warping can be included to satisfy the three-dimensional numerical analysis. As described above, the numerical result using the present element is slightly larger than the other two methods. The differences was caused by the mesh of the finite element mesh generation. The cable section can only be meshed by was meshed by one beam element, but by five or more degenerated solid elements. Therefore, the stress produced by the cable gravity can be computed and superimposed in the geometrical stiffness matrix using presented model.

\section{REFERENCES}

1. Enyuan Xiao \& H.M.Irvine. 1997. Characteristics of cable, Higway, (5):1-7 
2. Zhi Fang \& Zhiyong Zhang. 1997. Cable-stayed bridge cable force test, China Journal of Highway and Transport, (10)1:51-58.

3. H.M.Irvine. 1981. Cable Structure, MIT PRESS.

4. Jingfeng \& WangGuangyu Wu. 2007.Bridge structural simulation analysis theory and engineering application. Hangzhou. Zhejiang University Press.

5. Xing Xu \& Yong Gan. 2003. Degenerated solid plate element and its application in vibration analysis of plates, Journal of Rock Mechanics and Geotechnical Engineering . 